\title{
The cardiovascular effect of incretin-based therapies among type 2 diabetes: a systematic review and network meta-analysis
}

Shanshan $\mathrm{Wu}, \mathrm{PhD}^{1}$; Andrea Cipriani, $\mathrm{PhD}^{2}$; Zhirong Yang, $\mathrm{Master}^{3}$; Jun Yang, $\mathrm{MD}^{4}$; Ting Cai, $\mathrm{MD}^{4}$; Yang Xu, Master ${ }^{4}$; Xiaochi Quan, $\mathrm{MD}^{4}$; Yuan Zhang, Master ${ }^{5}$; Sanbao Chai, $\mathrm{PhD}^{6}$; Feng Sun, $\mathrm{PhD}^{4,7^{*}}$; Siyan Zhan, $\mathrm{PhD}^{4 *}$

1. National Clinical Research Center of Digestive Diseases, Beijing Friendship Hospital, Capital Medical University, Beijing, China, 100050.

2. Department of Psychiatry, University of Oxford, Oxford, UK; Oxford Health NHS Foundation Trust, Warneford Hospital, Oxford, UK.

3. Primary Care Unit, Department of Public Health and Primary Care, School of Clinical Medicine, University of Cambridge, Cambridge, UK, CB18RN.

4. Department of Epidemiology and Biostatistics, School of Public Health, Peking University Health Science Centre, Beijing, China, 100191.

5. Department of Clinical Epidemiology and Biostatistics, McMaster University, Hamilton, Canada, L8S4K1.

6. Department of Endocrinology and Metabolism, Peking University International Hospital, Beijing, China, 102206.

7. Department of Population Medicine, Harvard Medical School, Boston, USA, 02215

"Co-Corresponding author: Prof. Feng Sun, Department of Epidemiology and Biostatistics, School of Public Health, Peking University Health Science Centre, Beijing, China, 100191. Email: sunfeng@bjmu.edu.cn. Tel: 86-10-82801191-1055.

Prof. Siyan Zhan, Department of Epidemiology and Biostatistics, School of Public Health, Peking University Health Science Centre, Beijing, China, 100191. Email: siyan-zhan@ bjmu.edu.cn. Tel: 86-1082805162.

Word count of the text: 200 for abstract and 3321 for main text

NO. of references: 40

NO. of tables: 1

NO. of figures: 2

NO. of supplement material: 16 Appendix files. 


\section{ABSTRACT}

Objective: To evaluate the comparative cardiovascular safety of incretin-based therapies in patients with type 2 diabetes mellitus (T2DM).

Methods: Medline, Embase, the Cochrane Library and www.clinicaltrials.gov were searched for randomized controlled trials (RCTs) with duration $\geq 12$ weeks. Network meta-analysis was performed, followed by subgroup analysis and meta-regression. The Grading of Recommendations Assessment, Development and Evaluation system was used to assess the quality of evidence. The outcome of interest was a composite of cardiovascular death, myocardial infarction, stroke and heart failure. Odds ratio (OR) with $95 \%$ confidence interval (CI) was calculated as the measure of effect size.

Results: 281 RCTs (76.9\% double-blinded) with 180,000 patients were included, comparing incretinbased therapies with other six classes of anti-diabetic drugs or placebo. A statistically significant reduction in the risk of cardiovascular events was found in favour of GLP-1RAs when compared with placebo (OR 0.89, 95\%CI: 0.80-0.99) and sulfonylurea (OR 0.76, 95\%CI: 0.59-0.99), whereas DPP-4 inhibitors showed a neutral effect compared with placebo (OR 0.92, 95\%CI: 0.83-1.01).

Conclusions: Incretin-based therapies show similar cardiovascular risk in comparison with metformin, insulin, thiazolidinediones, alpha-glucosidase inhibitor and sodium-glucose co-transporter 2. GLP-1RA could decrease the risk compared with sulfonylurea or placebo, while DPP-4I appears to have neutral effect on cardiovascular risk.

Key words: Incretin-based therapies, type 2 diabetes, network meta-analysis, cardiovascular effect 


\section{Introduction}

Type 2 diabetes mellitus (T2DM) is a well-established risk factor for cardiovascular disease ${ }^{1}$. According to the results of the largest cohort study with 1.9 million subjects, T2DM was associated with a significantly increased risk of ischemic stroke (hazard ratio, $H R=1.72)$, stable angina $(H R=1.62)$, heart failure $(\mathrm{HR}=1.56)$ and non-fatal myocardial infarction $(\mathrm{HR}=1.54)$. Therefore, it is critical for antidiabetic drugs not to increase the risk of cardiovascular events. The US Food and Drug Administration has required proof of cardiovascular safety to approve new glucose-lowering drugs since the increased risk of myocardial infarction associated with rosiglitazone in $2008^{2}$.

Incretin-based therapies are a new class of anti-diabetic agents, including dipeptidyl peptidase-4 (DPP4) inhibitors and glucagon-like peptide-1 receptor agonists (GLP-1 RAs). DPP-4 inhibitors prevent the rapid degradation of GLP-1 through inhibition of DPP-4, thus enhancing pancreatic insulin secretion and suppress pancreatic glucagon secretion. GLP-1 RAs are analogues of GLP-1, which could stimulate insulin secretion, improve insulin resistance and slow down gastrointestinal motility ${ }^{3}$. Despite the increasing and widespread use of incretin-based therapies, substantial uncertainty about the cardiovascular safety still exists in these therapies.

So far there is lack of evidence comparing incretin-based therapies with other anti-diabetic treatments regarding cardiovascular safety in a trial network. The only one relevant network meta-analysis restricted incretin-based therapies to individual drugs of GLP-1 RAs ${ }^{4}$. While relevant pairwise meta-analyses have been available, they have not well demonstrated the cardiovascular safety issues of incretin-based therapies due to following limitations. Firstly, pairwise comparisons performed in these meta-analyses were mostly between DPP-4 inhibitors or GLP-1RAs and all active comparators combined in one class 5-18. This may potentially dilute the specific effect of incretin-based therapies and make the interpretation 
of results difficult from a clinical point of view. Secondly, some meta-analyses ${ }^{5-14,16-18}$ only included a limited number of trials focusing on one of the DPP-4 inhibitors or GLP-1RAs with relatively short trial duration (median follow-up of 1.5-3 years), leading to less statistical power for sparse-events data. Thirdly, clinical heterogeneity such age, trial duration, HbA1c\% level, and years of T2DM was not properly explored in most of the meta-analyses ${ }^{4-11}$. Additionally, a majority of these studies ${ }^{4-17}$ did not assess the quality of evidence and neglected its importance in explaining the results. All of these gaps restrain the evidence from fully informing clinical practice, making urgent need for high quality network meta-analysis.

Therefore, we aimed to collect all RCTs comparing incretin-based therapies with placebo or other antidiabetic drugs among patients with T2DM for at least 12 weeks and conduct a network meta-analysis to assess the comparative cardiovascular safety of individual classes of anti-diabetic agents. 


\section{Methods}

This study is registered with PROSPERO, number CRD42015020395.

\subsection{Data sources and Searches}

Medline, Embase and the Cochrane Central Register of Controlled Trials were searched from inception to April 28 $8^{\text {th }}, 2016$ (see Web Appendix 1 for full details about the search strategy). Clinical trial registries (such as www.clinicaltrials.gov) were searched for unpublished trials. In addition, we also checked the reference list of all relevant articles to identify additional studies.

\subsection{Study selection}

Only RCTs (either double-blind, single-blind or open-label) written in English and with available data on cardiovascular events in which incretin-based therapies (including DPP-4 inhibitors and GLP-1RAs) compared with other active drugs or placebo in patients with T2DM were included. The duration of trials was at least 12 weeks. For studies that were longer than 12 weeks, final endpoint data were used for the analysis. The outcome of interest was a composite of cardiovascular events, which consisted of cardiovascular death, myocardial infarction, stroke (the three major adverse cardiovascular events (MACEs) defined by the Food and Drug Administration ${ }^{19}$ ), and heart failure. For studies reporting adverse events but without specifically reporting cardiovascular events (zero-event in both arms), we also included them as zero-event in the analysis. The constant continuity correction method was used with an addition of a correction factor of 0.5 to the number of events and non-events in both treatment groups ${ }^{20}$. The eligibility of studies for inclusion criteria was assessed independently by four reviewers (SSW, JY, TC and FS) in duplicate. Any discrepancies were resolved by consensus between the two independent reviewers or by a senior investigator (FS).

\subsection{Data extraction and quality assessment}


Data were extracted using ADDIS software with respect to trial information (author, publication year, sample size, trial duration, types of intervention and control), population characteristics (background therapy, diabetes duration, age, baseline level of HbA1c), reported outcomes (number of cardiovascular events in each treatment group) and information on methodology. Four investigators (SSW, JY, TC and XCQ) extracted data independently, in duplicate. Risk of bias of included studies was assessed according to Cochrane risk of bias tool ${ }^{21}$. Additionally, the GRADE (The Grading of Recommendations Assessment, Development, and Evaluation) framework was used to assess the quality of evidence contributing to each network estimate, which characterizes the quality of a body of evidence on the basis of the study limitations, imprecision, inconsistency, indirectness and publication bias for the primary outcomes $^{22}$.

\subsection{Data Synthesis and Analysis}

\subsubsection{Methods for direct treatment comparisons}

Standard pairwise meta-analysis was performed using DerSimonian-Laird random effects model. Odds ratios (OR) for cardiovascular events with 95\% confidence interval (CI) were calculated as effect measures. The $I^{2}$-statistic was calculated for heterogeneity, as a measure of the proportion of the overall variation that is attributable to between-study heterogeneity. Besides, sensitivity analysis of pairwise meta-analysis was conducted to validate the robustness of the results by including double-blind studies and using only MACE as the outcome.

\subsubsection{Methods for indirect and mixed comparisons}

A random-effects network meta-analysis within a frequentist framework ${ }^{23}$ was then performed. OR for cardiovascular events with $95 \%$ CI was summarized. We estimated the ranking probabilities for all treatments of being at each possible rank for each intervention. The treatment hierarchy was summarized 
and reported as surface under the cumulative ranking curve (SUCRA) and mean ranks. SUCRA is a percentage interpreted as the probability of a treatment is the most effective without uncertainty on the outcome, which is equal to 1 when the treatment is certain to be the best and 0 when it is certain to be the worst. To check the assumption of consistency in the entire analytical network, a design-by-treatment approach was used ${ }^{24}$. A loop-specific approach was used to evaluate the presence of inconsistency locally in each closed loop. The node splitting method was used to assess the inconsistency of the model with separating evidence on a particular comparison into direct and indirect evidence. A global heterogeneity was assessed with $I^{2}$-statistic and predictive interval plot ${ }^{25}$ that incorporate the extent of heterogeneity was used to evaluate the extent of uncertainty in the estimated effect size locally. Uncertainty affected by heterogeneity was defined as disagreement between the confidence intervals of relative treatment effects and their predictive intervals. Contribution plot was used to assess the contribution of each direct comparison to the estimation of each network meta-analytic summary effect, since it was helpful to evaluate the overall quality of evidence from network meta-analysis ${ }^{25}$. Additionally, a comparison-adjusted funnel plot was used to detect the potential publication bias in the results between small and large studies. To assess whether the results were impacted by study characteristics (effect modifiers), subgroup analysis was conducted according to age group, trial duration, HbA1c\% level, years of T2DM, sample size, quality of study and sponsorship. Univariate and multivariate meta-regression was further conducted to control the confounding factors. Besides, sensitivity analysis of network meta-analysis was conducted to validate the robustness of the results by using only MACE as the outcome of interest and omitting open-label trials. All analyses were conducted using STATA 13.0 (pairwise meta-analysis, network meta-analysis, estimation of inconsistency and heterogeneity, funnel plot and SUCRA graphs) and R 3.3.0 (transforming data). 


\section{Results}

\subsection{Study characteristics}

Overall, 281 trials met the inclusion criteria (see Web appendix 2 for full reference list). Flow chart of trials selection was shown in Error! Reference source not found.. Eight treatments were analyzed, including incretin-based therapies (11 different DPP-4 inhibitors and 6 different GLP-1RAs), six other active anti-diabetic drugs [metformin, insulin, sulfonylurea, thiazolidinediones, alpha-glucosidase inhibitor (AGI) and sodium-glucose co-transporter 2(SGLT-2)] and placebo. 94.7\% (266/281) of trials were two-arm studies and only 15 were multiple-arm studies (Appendix 3). Overall, 180,000 patients contributed to the analysis of cardiovascular events (see Web appendix 4 for evidence network). Appendix 3 summarized the characteristics of the included trials. Publication year varied from 2004 to 2016. Trial duration ranged from 12 to 312 weeks with a median follow-up of 24 weeks [interquartile range (IQR): 18-48 weeks]. The mean age of included patients was 57.0 years [standard deviation (SD) 4.6], the median duration of diabetes at baseline was 6.7 years (IQR: 4.7-8.8) and the mean baseline HbA1c level was $8.1 \%$ (SD 0.6\%). Of the 281 trials included, DPP-4 inhibitors and GLP-1RAs were studies in 195 and 98 trials, respectively, and 12 trials involved both DPP-4 inhibitors and GLP-1RAs simultaneously. Among 195 trials including DPP-4 inhibitors, sitagliptin, vildagliptin, saxagliptin, linagliptin and alogliptin were the most commonly studied drugs, with 75, 45, 23, 23 and 19 trials, respectively. Out of the 98 RCTs on GLP-1RAs, exenatide, liraglutide, lixisenatide, albiglutide, taspoglutide, dulaglutide and semaglutide were studied in 35, 31, 12, 8, 7, 5 and 1 trial, respectively.

\subsection{Methodological quality and risk of bias results}

In terms of quality of included studies, allocation concealment and blinding of outcome assessment were not clearly reported in $15.0 \%$ and $67.5 \%$ of the cases, respectively. By contrast, the methods for 
randomization, blinding of participants and personnel and incomplete outcome data were appropriately described in the large majority of studies $(95.5 \%, 78.0 \%$ and $100 \%$, respectively). $21.7 \%(61 / 281)$ of trials were open label and $87.5 \%$ did not have selective reporting (the remaining $12.5 \%(35 / 281)$ was unclear due to no related protocol). Additionally, 89.3\% (251/281) of trials were funded by company and only $2.1 \%$ (6/281) did not report the funding sources (see Web appendix 5 for risk of bias assessment). Overall, the risk of bias across studies was relatively low.

\subsection{Results of pairwise meta-analysis}

Figure 2 showed the effects of incretin-based therapies and other active anti-diabetic drugs on cardiovascular events from pairwise meta-analyses. A total of 1,496 and 2,560 cardiovascular events developed in 30,962 and 70,401 patients with GLP-1RAs and DPP-4 inhibitors while 3,935 cardiovascular events occurred among 51,204 patients with placebo. GLP-1RAs were associated with a significant reduction of cardiovascular events compared with placebo (OR=0.89, 95\%CI: 0.82-0.69), while no significant difference was found when compared with other active comparators. DPP-4 inhibitors showed a neutral effect compared with placebo (OR=0.95, 95\%CI: 0.89-1.01) and a decreased risk compared with sulfonylurea $(\mathrm{OR}=0.69,95 \% \mathrm{CI}$ : $0.53--0.89)$. No statistically significant difference was found in terms of cardiovascular events between sulfonylurea (OR=0.65, 95\%CI: $0.22-1.94)$, thiazolidinediones $(\mathrm{OR}=0.84,95 \% \mathrm{CI}: 0.35$-2.01), SGLT-2 (OR=0.86, 95\%CI: $0.28-2.68)$ and placebo (see Web appendix 6 for pairwise analysis). As shown in Web appendix 7, sensitivity analysis of pairwise meta-analysis by using only MACE as the outcome of interest and including only double-blind trials confirmed the beneficial cardiovascular effect of GLP-1RAs in comparison to placebo or sulfonylurea.

\subsection{Results of network meta-analysis}

Results of the network meta-analysis are reported in Figure 2. For risk of cardiovascular events, the 
reduction was statistically significant for GLP-1RAs versus placebo (OR=0.89, 95\%CI: $0.80-0.99)$ and sulfonylurea (OR=0.76, 95\%CI: 0.59-0.99). The protective effect of GLP-1RAs was not observed in network meta-analysis compared with other active comparators [ranging between 0.72 (95\% CI: 0.45 1.14) for thiazolidinediones to 1.12 (95\% CI: 0.37-3.36) for AGI]. As for DPP-4 inhibitors, a neutral effect was detected when compared with placebo $(\mathrm{OR}=0.92,95 \% \mathrm{CI}$ : 0.83-1.01) and any active comparators. Besides, no significant association with cardiovascular events was found among any active comparators. According to the contribution plot of the network (see Web appendix 8), the comparison of placebo (treatment 1) versus GLP-1RAs (treatment 9) and DPP-4 inhibitors (treatment 2) had the largest contribution in the entire network (17.2\% and $19.9 \%$, respectively).

The test of global inconsistency did not detect any significant difference between the consistency and inconsistency models $(\mathrm{p}=0.368)$. Test for local inconsistency showed that most loops were consistent since their 95\% CIs included 1 according to the inconsistency plots (see Web appendix 9 for assessment of inconsistency). The common heterogeneity through the multivariate meta-analysis was 0.06 . The test of inconsistency from node-splitting model showed no significant difference in most comparisons, only three comparisons was with significant difference between direct and indirect comparisons (see Web appendix 9 for assessment of inconsistency). The global $I^{2}$ value was $0 \%$. Predictive interval plot indicated that none of the comparisons were substantially affected by the estimated heterogeneity in the network (see Web appendix 10 for assessment of heterogeneity). At visual inspection, funnel plot for cardiovascular events (see Web appendix 11 for comparison-adjusted funnel plot) was quite symmetric and did not suggest any significant risk of publication bias in our sample of included studies.

Table 1 showed the mean values of SUCRA for providing the hierarchy of 9 treatments on cardiovascular events (Web appendix 12 showed the ranking probabilities of each treatment). According 
to SUCRA, GLP-1RAs ranked first on decreasing cardiovascular risk among all 9 treatments with probability of $69.9 \%$. According to GRADE, the quality of evidence ranged between very low and moderate, but was rated as low for most comparisons, moderate for GLP-1RAs versus placebo and low for DPP-4 inhibitors versus placebo (see Web appendix 13 for contribution summary of risk of bias assessment and Web appendix 14 for quality of evidence according to GRADE framework). Quality of evidence was low for overall ranking of treatment for cardiovascular events (see Web appendix 14 for quality of evidence according to GRADE framework).

In addition, sensitivity analysis of network meta-analysis by using only MACE as the outcome of interest and including double-blind trials confirmed the beneficial cardiovascular effect of GLP-1RAs versus placebo and sulfonylurea, plus the neutral effect of DPP-4 inhibitors versus placebo, which were in agreement with those previous produced (see Web appendix 7 for sensitivity analysis). Subgroup analyses demonstrated that the beneficial cardiovascular effect of GLP-1RAs versus placebo was more evident in patients with $\mathrm{HbA} 1 \mathrm{c}$ level $\geq 8.5 \%$, DM duration $\geq 10$ years and older age (see Web appendix 15 for subgroup analysis). Univariate meta-regression indicated that the risk of cardiovascular events would decrease $33 \%$ for per $1 \%$ change of $\mathrm{HbA1c}$ (see Web appendix 16 for meta-regression analysis). Besides, findings of network meta-analysis only with trials at low risk of bias also confirmed the protective effect for GLP-1RAs and neutral effect for DPP-4 inhibitors when compared with placebo (see Web appendix 15 for subgroup analysis).

\section{Discussion}

Our network meta-analysis with 281 trials and 180,000 patients suggested that GLP-1RAs were associated with decreased risk of cardiovascular events compared with placebo and sulfonylurea, while DPP-4 inhibitors had a neutral effect on cardiovascular profiles in comparison with placebo and other 
traditional anti-diabetic drugs. Aside from adequate glycaemia control, increasing attention is being paid to the cardiovascular safety of incretin-based therapies recently. EXSCEL study also showed the incidence of major adverse cardiovascular events did not differ significantly between patients with exenatide and those with placebo ${ }^{26}$.

Four large cardiovascular trials ${ }^{27-30}$ for DPP-4 inhibitors and GLP-1RAs separately also failed to detect the favorable effect on cardiovascular events. The apparent inconsistency may be due to the limited sample size of those trials. The sample size of the four trials was $16,492,5,380,14,671$ and 6,068 , respectively, which was much smaller than the sample size of 180,000 patients in our analysis. As cardiovascular endpoint was a rare outcome, the limited sample size of each trial may be underpowered to assess the cardiovascular endpoint ${ }^{3,31}$. Moreover, the duration of those trials was relatively short (median follow-up of 1.5-3 years), making the beneficial cardiovascular effect not completely reflected 32. The United Kingdom Prospective Diabetes Study (UKPDS) and Diabetes Mellitus Insulin Glucose Infusion in Acute Myocardial Infarction (DIGAMI-1) trials all followed for up to 20-30 years to detect the significant reduction of cardiovascular risk in subjects initially treated with intensive glucoselowering therapy ${ }^{33,34}$. Therefore, the cardiovascular effect of incretin-based therapies may also require several years to achieve. Thirdly, participants in those trials were more complex multi-morbid with multifactorial interventions including anti-platelet, anti-hypertensive and lipid lowering agents, while patients included in our analysis were relatively healthier $(50 \%$ and $70 \%$ of the trials without antihypertensive and lipid lowering therapy, respectively) ${ }^{32}$. According to the comprehensive analysis of the effect of glycemic, blood pressure and cholesterol control on cardiovascular risk by Yudkin et al ${ }^{35}$, the number of subjects needed to treat for 5 years to prevent one cardiovascular event would be 44 with 1 $\mathrm{mmol} / \mathrm{l}$ cholesterol lowering, 34 with a 10/5 mmHg reduction in blood pressure, and 119 with intensive 
glucose lowering. Consequently, anti-hypertensive and lipid lowering treatments appear to have a greater impact than glucose lowering on cardiovascular events, making the favorable cardiovascular effect of incretin-based therapies less noticeable.

Nevertheless, several studies have supported the non-increasing effect of incretin-based therapies ${ }^{36-}$ 40. Both GLP-1RAs and DPP-4 inhibitors might exert a favorable cardiovascular effect through antiinflammatory mechanism, such as reductions in levels of tumor necrosis factor-alpha and mid-regional pro-adrenomedullin in patients with $\mathrm{T} 2 \mathrm{DM}{ }^{36}$. Moreover, incretin-based therapies have been shown to significantly reduce carotid intima-media thickness ${ }^{37}$, levels of albuminuria ${ }^{36,38}$, epicardial adipose tissue ${ }^{39,40}$ and several cardiovascular risk biomarkers ${ }^{36,37}$ in patients with T2DM. Thus it may reflect clinically relevant benefit in cardiovascular events. To date, several long-term prospective trials (see Web appendix 17) specially designed for cardiovascular outcomes of incretin-based therapies, such as CARMELINA (Cardiovascular and Renal Microvascular Outcome Study With Linagliptin in Patients With Type 2 Diabetes Mellitus, NCT01897532), are currently undergoing or in patients recruitment phase. Thus, it will take several years to confirm whether this protective effect on cardiovascular events, particularly for patients with higher HbA1c level, was true or not.

A major strength of our study is the comprehensive and substantial analysis of cardiovascular profiles of incretin-based therapies compared with placebo and other antidiabetic treatments separately in a whole trial network with high quality. In addition to the main analysis, we conducted detailed subgroup analyses and meta-regression by study characteristics (age group, trial duration, $\mathrm{HbA1c} \%$ level, years of T2DM, sample size, quality of study, sponsorship and classification of incretin-based therapies) to address the clinical heterogeneity of studies. Furthermore, we carried out sensitivity analyses by using only MACE as the outcome and including only double-blind trials, the results were consistent, which indicated that 
our findings were robust. Additionally, we assessed the quality of evidence and incorporate it into explaining the results by the GRADE framework.

Several limitations, however, should be mentioned and taken into account when interpreting the data from this study. First, most comparisons were assessed as low quality in the GRADE framework, which might restrict the interpretation of the results. Secondly, most trials included were not specially designed to evaluate the effect of incretin-based therapies on cardiovascular events. In nearly half of the trials, the outcome assessment (i.e. adjudication of cardiovascular events) was not blind and $90 \%$ of the studies were funded by the manufacturer of the investigational drug. Finally, we did not have access to original studies' data, so we could not perform an individual patient data meta-analysis to properly assess in our analyses potentially relevant effect modifiers such as, different baseline levels of diabetes duration and HbA1c or cardiovascular comorbidity (i.e. hypertension and hyperlipidemia).

\section{Conclusions}

GLP-1RAs not only show a similar risk of cardiovascular events in comparison with metformin, insulin, thiazolidinediones, alpha-glucosidase inhibitor and sodium-glucose co-transporter 2, but also appear to decrease the risk when compared with sulfonylurea or placebo, which seems to be a suitable option for long term treatment of type 2 diabetes mellitus. DPP-4 inhibitors seem to have a neutral effect when compared with placebo. However, the great majority of studies were funded by the pharmaceutical company marketing the investigational drug. Future guidelines should incorporate findings from this network meta-analysis, taking into account also the implications in terms of cost effectiveness for this new class of drugs.

\section{Acknowledgements}


We are grateful to all cooperating organizations and their staff whose hard work made this study possible. AC is supported by the NIHR Oxford Cognitive Health Clinical Research Facility. ZRY is supported by the Cambridge Trust and the China Scholarship Council.

\section{Funding}

This study is funded by National Natural Science Foundation of China $(81302508,71673003)$. The sponsor had no role in study design, data collection, data analysis, data interpretation, or writing of the report.

\section{Author Contributions}

FS and SYZ designed the study and drafted the manuscript. SSW, JY, TC and QXC extracted the data, SSW, ZRY, YZ and JY evaluated the RCTs quality. SSW, JY and FS assessed the quality of evidence by GRADE framework. TC, YX and ZRY verified the data, FS and SSW analyzed the data. FS, SYZ and AC interpreted the results, incorporated comments for the co-authors and finalized the manuscript. All authors approved the final version of the paper.

\section{Conflicts of interests}

All authors have completed the ICMJE uniform disclosure form at www.icmje.org/coi_disclosure.pdf and declare no potential conflicts of interest relevant to this article.

\section{References}

1. Shah AD, Langenberg C, Rapsomaniki E, Denaxas S, Pujades-Rodriguez M, Gale CP, et al. Type 2 diabetes and incidence of cardiovascular diseases: a cohort study in 1.9 million people. Lancet Diabetes Endocrinol. 2015; 3(2):105-13.

*2. US Food and Drug Administration. Guidance for Industry Diabetes Mellitus-Evaluating 
Cardiovascular Risk in New Antidiabetic Therapies to Treat Type 2 Diabetes. 2008. http://www.fda.gov/downloads/Drugs/GuidanceComplianceRegulatoryInformation/Guidances/ucm071 627. pdf. (accessed May 27, 2016).

A report indicating that cardiovascular risk is necessary for new antidiabetic therapies in patients with type 2 diabetes.

3. Nauck MA. Incretin therapies: highlighting common features and differences in the modes of action of glucagon-like peptide-1 receptor agonists and dipeptidyl peptidase-4 inhibitors. Diabetes Obes Metab 2016;18(3):203-16.

**4. Sun F, Yu K, Wu S, Zhang Y, Yang Z, Shi L, et al. Cardiovascular safety and glycemic control of glucagon-like peptide-1 receptor agonists for type 2 diabetes mellitus: a pairwise and network metaanalysis. Diabetes Res Clin Pract 2012; 98(3):386-95.

The only published evidence comparing incretin-based therapies with other anti-diabetic treatments regarding cardiovascular safety in a trial network.

5. Monami M, Dicembrini I, Nardini C, Fiordelli I, Mannucci E. Effects of glucagon-like peptide-1 receptor agonists on cardiovascular risk: a meta-analysis of randomized clinical trials. Diabetes Obes Metab 2014; 16(1):38-47.

6. White WB, Pratley R, Fleck P, Munsaka M, Hisada M, Wilson C, et al. Cardiovascular safety of the dipeptidyl peptidase-4 inhibitor alogliptin in type 2 diabetes mellitus. Diabetes Obes Metab 2013; 15(7):668-73.

7. Monami M, Ahre’n B, Dicembrini I, Mannucci E. Dipeptidyl peptidase-4 inhibitors and cardiovascular risk: a meta-analysis of randomized clinical trials. Diabetes Obes Metab 2013;15:112-20.

8. Wu S, Hopper I, Skiba M, Krum H. Dipeptidyl peptidase-4 inhibitors and cardiovascular outcomes: 
meta-analysis of randomized clinical trials with 55,141 participants. Cardiovasc Ther 2014; 32(4):14758.

9. Savarese G, Perrone-Filardi P, D'Amore C, Vitale C, Trimarco B, Pani L, et al. Cardiovascular effects of dipeptidyl peptidase-4 inhibitors in diabetic patients: a meta-analysis. Int J Cardiol 2015;181:239-44. 10. Marso SP, Lindsey JB, Stolker JM, House JA, Martinez Ravn G, Kennedy KF, et al. Cardiovascular safety of liraglutide assessed in a patient-level pooled analysis of phase 2: 3 liraglutide clinical development studies. Diab Vasc Dis Res 2011; 8(3):237-40.

11. Seshasai SR, Bennett RL, Petrie JR, Bengus M, Ekman S, Dixon M, et al. Cardiovascular safety of the glucagon-like peptide-1 receptor agonist taspoglutide in people with type 2 diabetes: an individual participant data meta-analysis of randomized controlled trials. Diabetes Obes Metab 2015; 17(5):50510.

12. Rosenstock J, Marx N, Neubacher D, Seck T, Patel S, Woerle HJ, et al. Cardiovascular safety of linagliptin in type 2 diabetes: a comprehensive patient-level pooled analysis of prospectively adjudicated cardiovascular events. Cardiovasc Diabetol 2015;14:57.

13. Iqbal N, Parker A, Frederich R, Donovan M, Hirshberg B. Assessment of the cardiovascular safety of saxagliptin in patients with type 2 diabetes mellitus: pooled analysis of 20 clinical trials. Cardiovasc Diabetol 2014;13:33.

14. McInnes G, Evans M, Del Prato S, Stumvoll M, Schweizer A, Lukashevich V, et al. Cardiovascular and heart failure safety profile of vildagliptin: a meta-analysis of 17,000 patients. Diabetes Obes Metab 2015; 17(11):1085-92.

15. Udell JA, Cavender MA, Bhatt DL, Chatterjee S, Farkouh ME, Scirica BM. Glucose-lowering drugs or strategies and cardiovascular outcomes in patients with or at risk for type 2 diabetes: a meta-analysis 
of randomised controlled trials. Lancet Diabetes Endocrinol 2015; 3(5):356-66.

16. Agarwal S, Parashar A, Menon V. Meta-analysis of the cardiovascular outcomes with dipeptidyl peptidase 4 inhibitors: validation of the current FDA mandate. Am J Cardiovasc Drugs 2014; 14(3):191207.

17. Monami M, Dicembrini I, Mannucci E. Dipeptidyl peptidase-4 inhibitors and heart failure: a metaanalysis of randomized clinical trials. Nutr Metab Cardiovasc Dis 2014; 24(7): 689-97.

18. Li L, Li S, Deng K, Liu J, Vandvik PO, Zhao P, et al. Dipeptidyl peptidase-4 inhibitors and risk of heart failure in type 2 diabetes: systematic review and meta-analysis of randomised and observational studies. BMJ 2016; 352: i610.

19. Nordisk Novo. Liraglutide (injection) for the Treatment of Patients with Type 2 Diabetes: NDA22341. Endocrine and Metabolic Drug Advisory Committee 2 April 2009. http://www.fda.gov/downloads/AdvisoryCommittees/Committees-

MeetingMaterials/Drugs/EndocrinologicandMetabolicDrugsAdvisoryCommittee/UCM148659.pdf. (accessed 1 March 2016).

20. Rücker G, Schwarzer G, Carpenter J, Olkin I. Why add anything to nothing? The arcsine difference as a measure of treatment effect in meta-analysis with zero cells. Stat Med 2009; 28(5):721-38.

21. Higgins JPT, Green S. Cochrane Handbook for Systematic Reviews of Interventions Version 5. 1. 0. The cochrane collaboration, 2011. http://handbook.cochrane.org/ (accessed April 27, 2016)

22. Salanti G, Del Giovane C, Chaimani A, Caldwell DM, Higgins JP. Evaluating the quality of evidence from a network meta-analysis. PLoS One 2014; 9(7): e99682.

23. Salanti G. Indirect and mixed-treatment comparison, network, or multipletreatments meta-analysis: Many names, many benefits, many concerns for the next generation evidence synthesis tool. Res Synth 
Methods 2012; 3(2): 80-97.

24. Higgins JP, Jackson D, Barrett JK, Lu G, Ades AE, White IR. Consistency and inconsistency in networkmeta-analysis: concepts and models for multi-arm studies. Res Synth Methods 2012; 3(2): 98110.

*25. Chaimani ASG. Visualizing assumptions and results in network meta-analysis: The network graphs package. Stata J 2015; 15: 905-50.

A report demonsrating the assumptions and statistical analysis for network meta-analysis.

26. Holman RR, Bethel MA, Mentz RJ, Thompson VP, Lokhnygina Y, Buse JB, et al. Effects of OnceWeekly Exenatide on Cardiovascular Outcomes in Type 2 Diabetes. N Engl J Med. 2017;377(13):12281239.

27. Zannad F, Cannon CP, Cushman WC, Bakris GL, Menon V, Perez AT, et al. Heart failure and mortality outcomes in patients with type 2 diabetes taking alogliptin versus placebo in EXAMINE: a multicentre, randomised, double-blind trial. Lancet 2015; 385(9982):2067-76.

28. Mosenzon O, Raz I, Scirica BM, Hirshberg B, Stahre CI, Steg PG, et al. Baseline characteristics of the patient population in the Saxagliptin Assessment of Vascular Outcomes Recorded in patients with diabetes mellitus (SAVOR)-TIMI 53 trial. Diabetes Metab Res Rev 2013; 29(5):417-26.

29. Green JB, Bethel MA, Paul SK, Ring A, Kaufman KD, Shapiro DR, et al. Rationale, design, and organization of a randomized, controlled Trial Evaluating Cardiovascular Outcomes with Sitagliptin (TECOS) in patients with type 2 diabetes and established cardiovascular disease. Am Heart J 2013; 166(6): 983-989.e7.

30. Pfeffer MA, Claggett B, Diaz R, Dickstein K, Gerstein HC, Køber LV, et al. Lixisenatide in Patients with Type 2 Diabetes and Acute Coronary Syndrome. N Engl J Med 2015; 373(23): 2247-57. 
31. Waldrop G, Zhong J, Peters M, Rajagopalan S. Incretin-Based Therapy for Diabetes: What a Cardiologist Needs to Know. J Am Coll Cardiol 2016; 67(12):1488-96. D

*32. Bolen SD, Maruthur NM. The safety of incretin based drug treatments for type 2 diabetes. BMJ 2016; 352: i801.

A important review relevant to the safety of incretin based drug treatments for patients with type 2 diabetes.

33. Hayward RA, Reaven PD, Wiitala WL. Follow-up of glycemic control and cardiovascular outcomes in type 2 diabetes. N Engl J Med 2015; 372(23): 2197-206.

*34. Ritsinger V, Malmberg K, Mårtensson A, Rydén L, Wedel H, Norhammar A. Intensified insulinbased glycaemic control after myocardial infarction: mortality during 20 year follow-up of the randomised Diabetes Mellitus Insulin Glucose Infusion in Acute Myocardial Infarction (DIGAMI 1) trial. Lancet Diabetes Endocrinol 2014; 2(8): 627-33.

A study indicating the cardiovascular effect of incretin-based therapies may also require several years to achieve.

35. Yudkin JS, Richter B, Gale EA. Intensified glucose lowering in type 2 diabetes: time for a reappraisal. Diabetologia 2010; 53(10): 2079-85.

36. von Scholten BJ, Persson F, Rosenlund S, Eugen-Olsen J, Pielak T, Faber J, et al. Liraglutide effects on cardiovascular risk biomarkers in patients with type 2 diabetes and albuminuria: A sub-analysis of a randomised, placebo-controlled, double-blind, cross-over trial Diabetes Obes Metab 2017; 19(6): 901905.

37. Rizzo M, Rizvi AA, Patti AM, Nikolic D, Giglio RV, Castellino G, et al. Liraglutide improves metabolic parameters and carotid intima-media thickness in diabetic patients with the metabolic 
syndrome: an 18-month prospective study. Cardiovasc Diabetol 2016; 15(1):162.

38. Mosenzon O, Leibowitz G, Bhatt DL, Cahn A, Hirshberg B, Wei C, et al. Effect of Saxagliptin on Renal Outcomes in the SAVOR-TIMI 53 Trial. Diabetes Care 2017; 40(1):69-76.

39. Dutour A, Abdesselam I, Ancel P, Kober F, Mrad G, Darmon P, et al. Exenatide decreases liver fat content and epicardial adipose tissue in patients with obesity and type 2 diabetes: a prospective randomized clinical trial using magnetic resonance imaging and spectroscopy. Diabetes Obes Metab 2016; 18(9):882-91.

40. Iacobellis G, Mohseni M, Bianco SD, Banga PK. Liraglutide causes large and rapid epicardial fat reduction. Obesity (Silver Spring) 2017; 25(2):311-316. 
Table 1. Ranking probability of different kinds of glucose-lowering drugs on cardiovascular events

\begin{tabular}{llc}
\hline & \multicolumn{2}{c}{ Cardiovascular events } \\
\cline { 2 - 3 } Treatment & SUCRA & Rank \\
\hline Placebo & 0.414 & 7 \\
DPP-4I & $\mathbf{0 . 6 2 9}$ & $\mathbf{3}$ \\
GLP-1RA & $\mathbf{0 . 6 9 9}$ & $\mathbf{1}$ \\
Insulin & 0.596 & 5 \\
Met & 0.501 & 6 \\
SGLT-2 & 0.600 & 4 \\
SU & 0.217 & 8 \\
AGI & $\mathbf{0 . 6 3 2}$ & $\mathbf{2}$ \\
TZD & 0.212 & 9
\end{tabular}

Note: Ranking: probability of being the best treatment, of being the second best, the third best and so on, among the 8 treatments. SUCRA: surface under the cumulative ranking curve. DPP-4I: dipeptidyl peptidase-4 inhibitors; GLP-1RA: glucagon-like peptide-1 receptor agonists; SGLT-2: sodium-glucose co-transporter 2; Met: metformin; SU: sulphanylureas; AGI: alpha-glucosidase inhibitor; TZD: thiazolidinediones. 


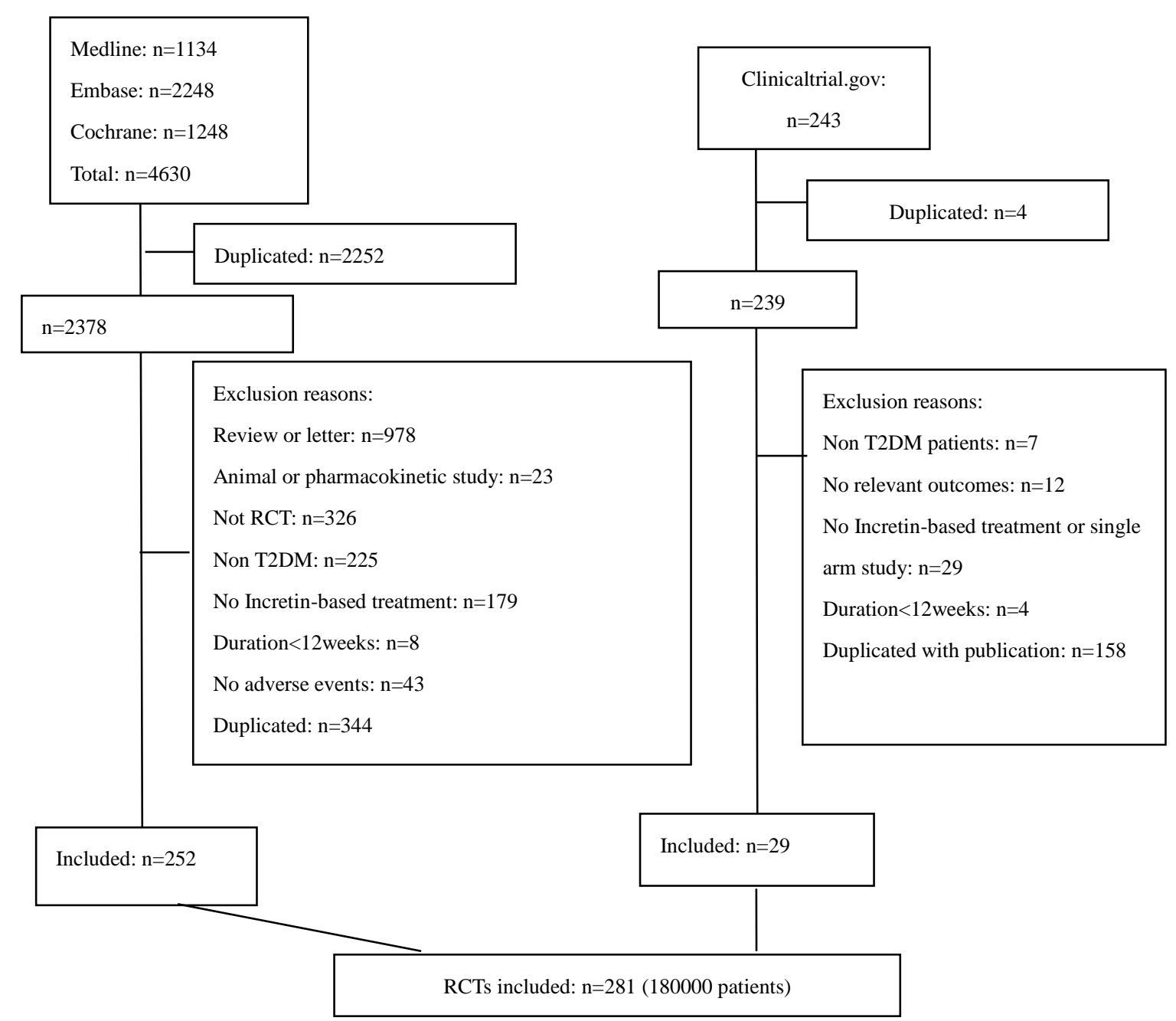

Figure 1. Flow chart of studies considered for inclusion. RCT: randomized controlled trial. 


\begin{tabular}{|c|c|c|c|c|c|c|c|c|}
\hline DPP-4I & $0.91(0.49,1.67)$ & $\begin{array}{l}0.75(0.18 \\
3.03)\end{array}$ & $0.75(0.27,2.05)$ & $1.03(0.40,2.63)$ & $0.69(0.53,0.89)$ & $1.15(0.39,3.44)$ & $0.97(0.52,1.82)$ & $\begin{array}{l}0.95(0.89, \\
1.01)\end{array}$ \\
\hline $\begin{array}{l}1.03(0.90,1.18 \\
)\end{array}$ & GLP-1RA & $\begin{array}{l}1.03 \\
(0.65,1.64)\end{array}$ & $\begin{array}{l}0.69 \\
(0.14,3.38) \\
\end{array}$ & $\begin{array}{l}1.03 \\
(0.40,2.63) \\
\end{array}$ & $\begin{array}{l}1.38 \\
(0.82,2.32) \\
\end{array}$ & $\begin{array}{l}1.15 \\
(0.39,3.45) \\
\end{array}$ & $0.29(0.12,0.66)$ & \begin{tabular}{|l|}
0.89 \\
$(0.82,0.96)$ \\
\end{tabular} \\
\hline $\begin{array}{l}1.02(0.65,1.59 \\
)\end{array}$ & $0.99(0.64,1.52)$ & Insulin & NA & NA & NA & NA & NA & $1.27(0.21,7.78)$ \\
\hline $\begin{array}{l}0.94(0.42,2.09 \\
)\end{array}$ & $0.91(0.40,2.04)$ & $\begin{array}{l}0.92 \\
(0.37,2.30)\end{array}$ & Met & NA & NA & NA & NA & NA \\
\hline $\begin{array}{l}1.05(0.48,2.33 \\
)\end{array}$ & $1.02(0.46,2.28)$ & $\begin{array}{l}1.04 \\
(0.42,2.57) \\
\end{array}$ & $\begin{array}{l}1.13 \\
(0.36,3.48) \\
\end{array}$ & SGLT-2 & NA & NA & NA & \begin{tabular}{|l|}
0.86 \\
$(0.28,2.68)$ \\
\end{tabular} \\
\hline $\begin{array}{l}0.79(0.62,1.00 \\
)\end{array}$ & $0.76(0.59,0.99)$ & $\begin{array}{l}0.77 \\
(0.47,1.27) \\
\end{array}$ & $\begin{array}{l}0.84 \\
(0.36,1.94) \\
\end{array}$ & $\begin{array}{l}0.75 \\
(0.33,1.71) \\
\end{array}$ & SU & NA & NA & $\begin{array}{l}0.65 \\
(0.22,1.94) \\
\end{array}$ \\
\hline $\begin{array}{l}1.15(0.39,3.44 \\
)\end{array}$ & $1.12(0.37,3.36)$ & \begin{tabular}{|l|}
1.13 \\
$(0.35,3.70)$ \\
\end{tabular} & $\begin{array}{l}1.23 \\
(0.32,4.78) \\
\end{array}$ & $\begin{array}{l}1.09 \\
(0.28,4.22) \\
\end{array}$ & $\begin{array}{l}1.47 \\
(0.48,4.50) \\
\end{array}$ & AGI & NA & NA \\
\hline $\begin{array}{l}0.74(0.47,1.17 \\
)\end{array}$ & $0.72(0.45,1.14)$ & $\begin{array}{l}0.73 \\
(0.39,1.36) \\
\end{array}$ & $\begin{array}{l}0.79 \\
(0.33,1.90) \\
\end{array}$ & $\begin{array}{l}0.70 \\
(0.28,1.75) \\
\end{array}$ & $\begin{array}{l}0.94 \\
(0.56,1.58) \\
\end{array}$ & $\begin{array}{l}0.64 \\
(0.20,2.11) \\
\end{array}$ & TZD & $\begin{array}{l}0.84 \\
(0.35,2.01) \\
\end{array}$ \\
\hline $\begin{array}{l}0.92(0.83,1.01 \\
)\end{array}$ & $0.89(0.80,0.99)$ & $\begin{array}{l}0.90 \\
(0.58,1.40)\end{array}$ & $\begin{array}{l}0.98 \\
(0.44,2.20)\end{array}$ & $\begin{array}{l}0.87 \\
(0.39,1.93)\end{array}$ & $\begin{array}{l}1.17 \\
(0.91,1.51)\end{array}$ & $\begin{array}{l}0.80 \\
(0.27,2.39)\end{array}$ & $1.24(0.78,1.97)$ & Placebo \\
\hline
\end{tabular}

Figure 2. Odds ratio $(\mathrm{OR})$ with $95 \% \mathrm{CI}$ of network meta-analysis for cardiovascular events.

Note: Treatments were reported in alphabetical order. Results of direct comparisons were listed in the upper triangle, and the estimation was calculated as the row-defining treatment compared with the column-defining treatment. Results of network meta-analysis were listed in the lower triangle, and the estimation was calculated as the columndefining treatment compared with the row-defining treatment. NA: not available. DPP-4I: dipeptidyl peptidase-4 inhibitors; GLP-1RA: Glucagon-like peptide-1 receptor agonists; SGLT-2: Sodium-Glucose co-Transporter 2; Met: metformin; SU: sulphanylureas; AGI: alpha-glucosidase inhibitor; TZD: thiazolidinediones. 